\title{
Time-gated CMOS SPAD and a Quantum Well Laser Diode with a CMOS Driver for Time-Resolved Diffuse Optics Imaging
}

\author{
J. Nissinen, I. Nissinen, S. Jahromi, T. Talala and J. Kostamovaara \\ University of Oulu, Circuits and Systems Research Unit \\ Oulu, Finland \\ jan.nissinen@oulu.fi
}

\begin{abstract}
Single-Photon Avalanche Photodiodes (SPADs) were fabricated and characterized in $150 \mathrm{~nm}$ CMOS technology. The SPAD is based on a p+/nwell junction with a p-substrate guard ring. In addition, a compact gain switched quantum well (QW) laser diode with a CMOS driver was used with the proposed SPAD for time-resolved diffuse optics measurements. The measured impulse response function (IRF) of the SPADs was $\sim 50$ ps at best. Two phantoms were measured to demonstrate the suitability of SPADs for time-resolved diffuse optics imaging (TRDOI).
\end{abstract}

Keywords-Impulse response function, Time-resolved diffuse optics, Single-photon avalanche diode

\section{INTRODUCTION}

During the last ten years single-photon avalanche diodes (SPADs) and diode arrays with timing electronics have been introduced in many kind of applications such as fluorescence lifetime imaging, time-of-flight (TOF) laser range imaging, positron emission tomography, time-resolved diffuse optical imaging/spectroscopy (TRDOI) and time-gated Raman spectroscopy [1-5]. One of the key factors in the rapid development of these applications has been the possibility for integrating both SPADs and timing electronics into the same CMOS chip, resulting in a high integration level and smaller size. Most of the above applications require sub-nanosecond timing accuracy.

Diffuse optical imaging is used for the characterization of diffusive media such as biological tissues, wood or chemical compounds [1]. In the wavelength range of 600 to $1000 \mathrm{~nm}$ the absorption of most biological media is low and the predominant interaction between the matter and the photons is caused by scattering, in which case photons can travel deeper inside the media. In the traditional continuous-wave (CW)-based system architecture the absolute values for the absorption $\left(\mu_{\mathrm{a}}\right)$ and reduced scattering coefficient $\left(\mu_{\mathrm{s}}^{\prime}\right)$ cannot be defined because measurement relies only on a change in the light intensity [6]. In addition, in order to probe deeper into the tissue the distance between the source-detector pair has to be increased when a $\mathrm{CW}$ system is used. Also, such systems suffer from low spatial resolution caused by the interaction between light absorption and scattering. In addition, they are very sensitive to motion artifacts and suffer from limited information content. Contrary to $\mathrm{CW}$ systems, time-resolved techniques enable the simultaneous evaluation of both absorption $\left(\mu_{\mathrm{a}}\right)$ and the reduced

This work was supported by Academy of Finland, Centre of Excellence in Laser Scanning Research under Contract 307362 and Contracts 292609, 282405 and 314404. scattering coefficients $\left(\mu_{\mathrm{s}}{ }^{\prime}\right)$ from a single measurement because both coefficients affect the photon distribution differently at early and late detection times [7]. Furthermore, information from different depths in the tissue is directly resolvable from the time of flight of the path length of the detected photons.

In time-resolved diffuse optical imaging (TRDOI) the distance between the source and detector should be kept as short as possible, or else even a null distance could be used to maximize the signal level, because information from deeper in the tissue can be resolved from the photons which arrive at the detector later in time [8]. The impulse response function (IRF) of the laser and the time-of-arrival distribution (DToF) of the photons, together with the measurement principle of the TRDOI system, are presented in Figs. 1 a) and b), respectively. For example, the photon distribution in healthy tissue is shown in Fig.1 a) (dotted line curve). The late photons probe deeper into the tissue, as marked with colours. Thus, in case of a tumour, which will increase the absorption coefficient, less detected photons will arrive at the detector after the path length time of photons in a proportion relative to depth of the tumour from the surface, as clarified in Figs.1 a) and b).

From the detector point of view, there has been a growing interest in using time-gated single-photon avalanche diodes (SPADs) or an array of diodes as a detector instead of a commercially available silicon photomultiplier (SiPM). A SPAD is a photodiode which is biased over the breakdown voltage, allowing an avalanche breakdown to be triggered even by a single photon. The inherent timing resolution of a SPAD is approximately $50-100$ ps when fabricated in CMOS technologies, which matches well with the timing requirements of this application. As mentioned above, the timing electronics can be integrated into the same CMOS chip, thus allowing a high integration level. It also makes it possible to use time-gated detection with late photons instead of an overwhelming burst of early photons, resulting in a possibility for probing deeper into the tissue. In addition, time-to-digital converters realized in CMOS technologies can achieve a resolution and accuracy of less than a few tens of picoseconds when integrated together with SPAD arrays $[9,10]$.

From the application point of view, the size of the laser source has been a bottleneck in constructing a small, compact optode or TRDOI prototype. Usually pulsed diode laser or Vertical-Cavity Surface-Emitting Laser (VCSEL) techniques are used when aiming at small size and low cost $[8,11]$, but the 
possibility for fabricating a small wearable optode is still limited by the size of the driver. In reference [12] a gain-switched quantum well (QW) laser diode with a CMOS driver approximately couple of cubic centimetres in size was proposed for TRDOI measurements with a commercial SiPM. This laser diode structure also allowed different wavelengths to be produced, thus permitting multispectral measurements [13,14]. To construct a compact measurement device or wearable optode for TRDOI measurements, the integration level of both the semiconductor laser diode driver and the time-gated SPAD array with timing electronics should be increased, which is possible using CMOS technology.

In this work, several time-gated SPAD structures were fabricated in $150 \mathrm{~nm}$ CMOS technology. They were then characterized and a CMOS driver-based gain-switched quantum well (QW) laser diode was used to demonstrate their suitability for a TRDOI application.

\section{TEST CHIP STRUCTURE}

The test chip was fabricated in a $150 \mathrm{~nm}$ CMOS technology. The SPAD is based on a p+/nwell active area junction, as shown in Fig. 2 a), where the cross-section of the SPAD is presented. The guard ring of the SPAD is obtained by blocking both the nwell and the pwell at the borders of the junction to prevent the SPAD from undergoing premature edge breakdown. The doping of the guard ring is close to the p-type substrate in this case. An $\mathrm{n}$-isolayer is formed below the active area to connect the nwell under $\mathrm{p}+$ to a cathode contact next to the guard ring [15]. Three different shapes of SPAD (circular, octagonal and capsular) were designed, as shown in Fig. 2 b), and their sizes were selected so that the octagonal ones were $10 \mu \mathrm{m}, 20 \mu \mathrm{m}$ and 40 $\mu \mathrm{m}$ in size, the circular ones were $20 \mu \mathrm{m}$ in diameter and the capsular ones were $10 \mu \mathrm{m}$ x $40 \mu \mathrm{m}$ and $10 \mu \mathrm{m}$ x $100 \mu \mathrm{m}$. Three SPADs of each shape and size were fabricated to guarantee that at least one good sample of each was achieved.

The front-end circuit of each SPAD and the timing diagram of the control signals are shown in Fig. 3. The operation of the front-end circuits is as follows. At the beginning the anode of the SPAD is in the position $\mathrm{Vdd}$, because both $\mathrm{V}_{\mathrm{Q}}$ and $\mathrm{V}_{\mathrm{L}}$ are 0 $\mathrm{V}$. Reverse bias of the SPAD ( $\left.\mathrm{V}_{\mathrm{HV}}\right)$ is selected so that the breakdown voltage $\mathrm{V}_{\mathrm{BR}}<\mathrm{V}_{\mathrm{HV}}-\mathrm{V}_{\mathrm{dd}}$, in which case the SPAD is self-quenched when the anode rises to $V_{d d}$. The difference between $V_{\mathrm{HV}}$ and $\mathrm{V}_{\mathrm{BR}}$ is the excess bias of the SPAD. In the next phase $\mathrm{V}_{\mathrm{Q}}$ is set to $\mathrm{V}_{\mathrm{DD}}$ and the anode is left floating. Immediately after that $V_{L}$ is set to $V_{D D}$ for a short time so that the NMOS transistor discharges the anode to ground, in which case the voltage over the SPAD is greater than the breakdown voltage and it is ready to detect photons. Both M1 and M2 are $5 \mathrm{~V}$ transistors, so that excess bias voltages higher than $1.8 \mathrm{~V}$ can be used, this being the logic level of the buffer chain. In addition, a $5 \mathrm{~V}$ clamp transistor (M3) is included between the anode and the first inverter to avoid oxide breakdown of the $1.8 \mathrm{~V}$ inverter. The first inverter connected to this clamp transistor acts as a comparator and was designed so that its threshold was approximately $0.8 \mathrm{~V}$. All the SPADs can be measured individually by enabling a single SPAD by means of a four-bit digital control word.

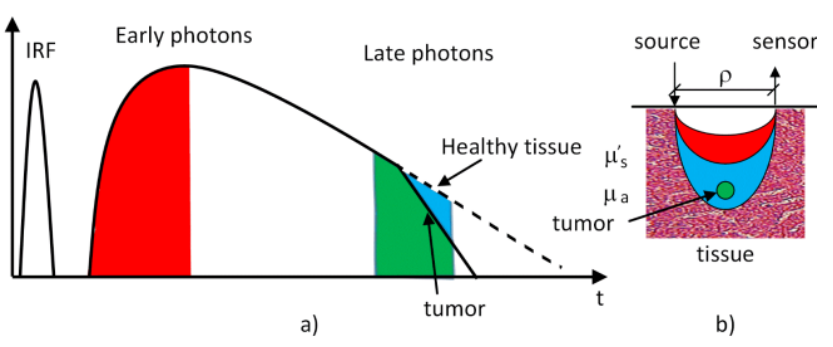

Fig. 1. a) IRF of the laser and distribution of the time-of-flight in a TRDOI measurement, and b) the principle of TRDOI measurement.

\section{ChARACTERIZATION MEASUREMENTS}

To measure the time-of-arrival of the dark counts or photons in the IRF measurements of the SPADs, start and stop signals for an off-chip time-to-amplitude converter (ORTEC TAC 566) were generated by a single-to-differential inverter as shown in Fig. 3 a). The start event for the TAC is generated by the inverter when the anode node is discharged to ground by $\mathrm{V}_{\mathrm{L}}$ (i.e. the SPAD is ready to detect photons). The stop signal is produced by a photon or dark count generating the rising edge at the output of the single-to-differential inverter. If the SPAD is not triggered by any photons or dark counts it will eventually be quenched by the PMOS transistor controlled by $\mathrm{V}_{\mathrm{Q}}$, as shown in Figs. 3 a) and $b$ ). The length of $\mathrm{V}_{\mathrm{Q}}$ defines the time gate window. A gate window of length $68 \mathrm{~ns}$ was used in all the measurements. After quenching a new measurement cycle can be started.

\section{A. Breakdown voltage mesurements}

The breakdown voltages of the SPADs were evaluated with active structures by monitoring output counts when increasing the reverse bias. A pulse generator was used to generate the control signals $\mathrm{V}_{\mathrm{L}}$ and $\mathrm{V}_{\mathrm{Q}}$ for actively loaded and quenched SPADs.

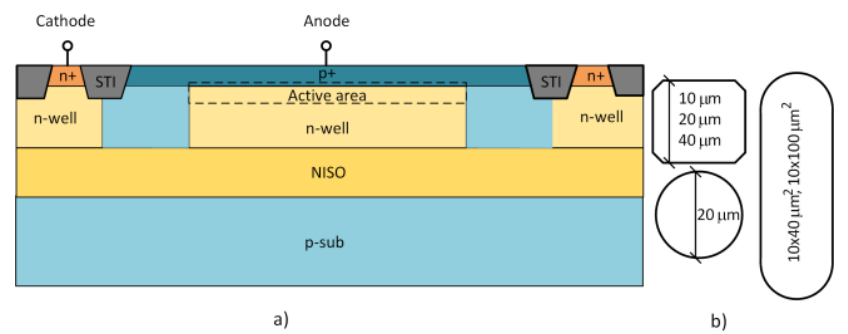

Fig. 2. a) Cross-section of a SPAD, and b) shapes of the SPADs.
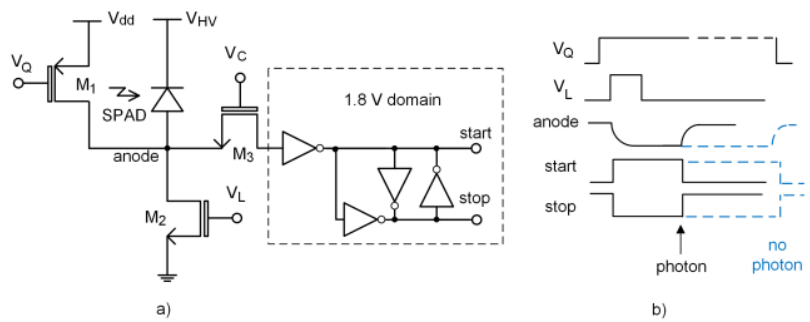

b)

Fig. 3. a) Front-end structure of a SPAD, and b) timing diagrams of the control signals and outputs. 
In the breakdown voltage measurements the selected SPAD was enabled for $68 \mathrm{~ns}$ before being quenched and the times-ofarrival of dark counts were observed by the TAC. The breakdown voltage was evaluated at the point where the TAC started to be triggered by dark counts or background noise. Note that a low background intensity is needed to see when the SPAD starts to breakthrough when working at a very low excess bias in an actively quenched structure where a time-gate of $68 \mathrm{~ns}$ is being used. The measured breakdown voltages of the SPADs in all the structures were approximately $18.7 \mathrm{~V}$.

\section{B. Dark count rate measurements}

Three excess bias voltages were used in the dark count rate (DCR) measurements: $1 \mathrm{~V}, 2.5 \mathrm{~V}$ and $3.5 \mathrm{~V}$. The measurements were carried out in gated operation, so that the SPAD was enabled over breakdown for a period of $68 \mathrm{~ns}$ at a pulsing rate of $30 \mathrm{kHz}$ and the dark counts were measured by the TAC. Measurement was continued until 1e5 counts $(\mathrm{N})$ were reached. The DCRs, expressed in $\mathrm{Hz}$, were calculated according to the total enabling time of the SPAD and the counts measured by the TAC, according to (1):

$$
D C R \approx \frac{N}{68 n s \cdot f_{\text {rate }} \cdot \Delta T}
$$

where $\Delta \mathrm{T}$ is the measurement time, $\mathrm{f}_{\text {rate }}$ is the repetition rate of the laser and $68 \mathrm{~ns}$ is the time-gate window.

The results are shown in Table 1, where averages for three similar SPADs are calculated. As can be seen, the DCR increases with the area of the SPAD. The measurements were carried out at room temperature.

\section{Impulse response function (IRF) measurements}

A gain a switched QW laser diode was used as a source in the jitter measurements [14]. The optical pulse of the laser diode was used to illuminate SPADs via optical fibres and a neutral density filter was employed to attenuate the optical power so that a photon detection probability of less than $5 \%$ was achieved. The time-of-arrival of the photons was measured by the TAC to form the distribution of the time-of-flight (DToF). The measured IRF is a convolution of the laser pulse, the IRF of the SPAD and the single-shot precision of the TAC (15 ps), in which case the IRF of the SPAD can be evaluated by subtracting the IRF of the laser and the single-shot precision of the TAC from the measured histogram. A pin diode receiver with a bandwidth of $25 \mathrm{GHz}$ and a $12 \mathrm{GHz}$ oscilloscope was used to measure the width of the optical pulse, which was 80 ps (FWHM) at the wavelength of $800 \mathrm{~nm}$. The optical pulse and the total IRF of the measurement setup with different SPADs are shown in Fig. 4. The IRFs of the SPADs were calculated from the time-of-arrival results according to (2). These results are shown in Table 2.

$$
I R F_{S P A D} \approx \sqrt{I R F_{\text {meas. }}^{2}-I R F_{L D}^{2}-I R F_{T A C}^{2}}
$$

\section{PROOF-OF-CONCEPT MEASUREMENT OF TRDOI}

To demonstrate the possibility for using the proposed SPADs with the QW laser diode driven by a CMOS driver for TRDOI measurements, two turbid polyurethane phantoms (india ink and titanium dioxide added) of different kinds (P1 and P2) were measured with the setup shown in Fig. 5 a). The laser diode with driver and the SPAD test IC are shown in Fig. $5 \mathrm{~b})$. A pulse generator was used to trigger the laser and simultaneously to generate loading and quenching pulses for the SPAD and to adjust the time-gate window so that photons from a turbid media could be detected. The repetition rate of the laser was set to $100 \mathrm{kHz}$, and a TAC was used to measure the time-of-arrival (TOA) of the photons and produce the distribution of time-of-flight (DToF). The absorption and reduced scattering coefficients of the phantoms $\mathrm{P} 1$ and $\mathrm{P} 2$ were $0.03 \mathrm{~mm}^{-1}$ and $0.8 \mathrm{~mm}^{-1}$ and $0.06 \mathrm{~mm}^{-1}$ and $0.29 \mathrm{~mm}^{-1}$, respectively. An octagonal SPAD of size $20 \mu \mathrm{m}$ was used in the TRDOI measurements.

TABLE I. DARK COUNT RATES OF THE SPADS AT THREE LEVELS OF

\begin{tabular}{c|c|c|c|c|c|c|} 
& $\begin{array}{c}10 \\
\mu \mathrm{m}\end{array}$ & $\begin{array}{c}20 \\
\mu \mathrm{m}\end{array}$ & $\begin{array}{c}40 \\
\mu \mathrm{m}\end{array}$ & $\begin{array}{c}10 \times 40 \\
\mu \mathrm{m}^{2}\end{array}$ & $\begin{array}{c}10 \times 100 \\
\mu \mathrm{m}^{2}\end{array}$ & $\begin{array}{c}20 \mu \mathrm{m} \\
\text { circle }\end{array}$ \\
\hline DCR 1 V kHz & 8.4 & 23 & 94 & 33 & 59 & 27 \\
DCR 2.5 V kHz & 12 & 32 & 126 & 51 & 83 & 38 \\
DCR 3.5 V kHz & 28 & 59 & 218 & 139 & 164 & 70 \\
\hline
\end{tabular}

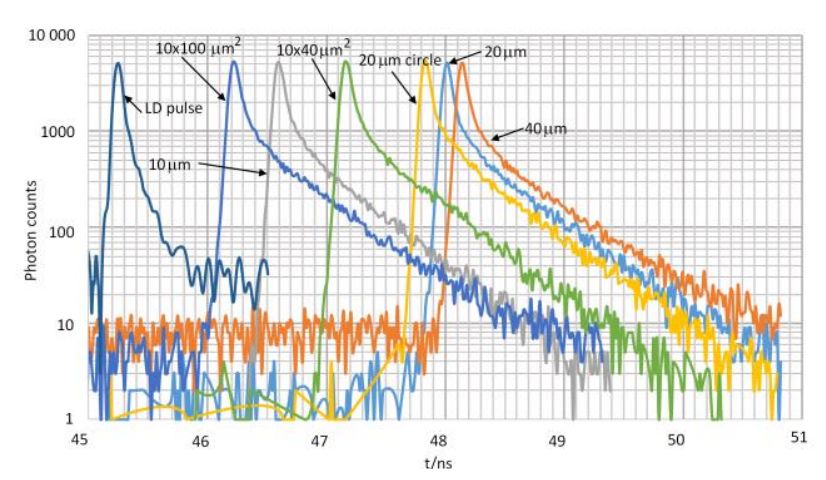

Fig. 4. Total single-shot precision of the various SPADs and the optical pulse.

TABLE II. CALCULATED FULL WIDTH HALF MAXIMUM OF THE IRFS OF THE VARIOUS SPADS

\begin{tabular}{c|c|c|c|c|c|c|} 
& $\begin{array}{c}10 \\
\mu \mathrm{m}\end{array}$ & $\begin{array}{c}20 \\
\mu \mathrm{m}\end{array}$ & $\begin{array}{c}40 \\
\mathrm{~mm}\end{array}$ & $\begin{array}{c}10 \times 40 \\
\mu \mathrm{m}^{2}\end{array}$ & $\begin{array}{c}10 \times 100 \\
\mu \mathrm{m}^{2}\end{array}$ & $\begin{array}{c}20 \mu \mathrm{m} \\
\text { circle }\end{array}$ \\
\hline FWHM of SPAD & $60 \mathrm{ps}$ & $50 \mathrm{ps}$ & $54 \mathrm{ps}$ & $70 \mathrm{ps}$ & $67 \mathrm{ps}$ & $48 \mathrm{ps}$ \\
\hline
\end{tabular}

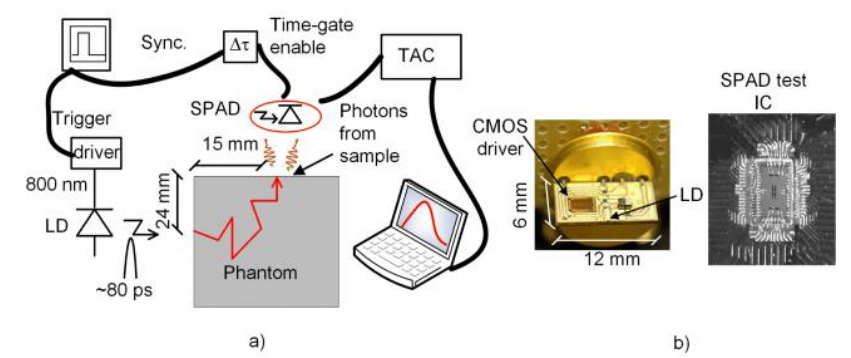

Fig. 5. a) Measurement setup for TRDOI, and b) photographs of the laser diode driver PCB and the SPAD test IC. 
Both of the phantoms were measured as shown in Fig. 5 a), ie. the laser diode and the detector were placed on adjacent surfaces of the phantom. The distance of the laser diode from the surface where the detector was placed was $24 \mathrm{~mm}$ and the detector was $15 \mathrm{~mm}$ from the surface of the laser diode, as shown in Fig. 5 a). Both the IRF of the laser pulse with the SPAD and the DToF of the photons detected through phantoms P1 and $\mathrm{P} 2$ are shown in Fig. 6. As can be seen in the DToF of the phantoms, the FWHM of the DToF of P2 (which has a larger absorption coefficient) is shorter, because the photons are absorbed in the phantom at a shorter absorption length so that they do not reach the detector in the late times as often as with P1 (which has a smaller absorption coefficient). On the other hand, P2 has a smaller reduced scattering coefficient, in which case photons arrive at the detector earlier, because they do not scatter as often in their path length. The measured FWHM of the DToFs of phantoms 1 and 2 and the IRF of the SPAD with laser used here were 430 ps, 300 ps and 90 ps, respectively.

\section{CONCLUSIONS}

A lot of research has been carried out during the last decade on the fabrication of a small-sized, low cost TRDOI system with simple structure. We have reported here preliminary test measurements performed on time-gated SPAD structures fabricated in $150 \mathrm{~nm}$ CMOS technology. The IRF and DCR of the $20 \mu \mathrm{m}$ octagonal SPAD used in the TRDOI measurements were approximately $50 \mathrm{ps}$ and $32 \mathrm{kHz}$, respectively, at an excess bias of $2.5 \mathrm{~V}$.

In addition, two phantoms were measured to demonstrate the suitability of the time-gated SPAD and the gain-switched QW laser diode driven by a small-sized CMOS driver for TRDOI measurement. The size and complexity of the system in these proposed structures could still be reduced, however, which in principle would make it possible to fabricate a multichannel TRDOI system in which all the key components (sensor and driver of the laser) were integrated in CMOS technology. Integration of the timing electronics into the same IC as the timegated SPADs would then pave the way to constructing a small, wearable optode for applications in the medical and health care sectors.

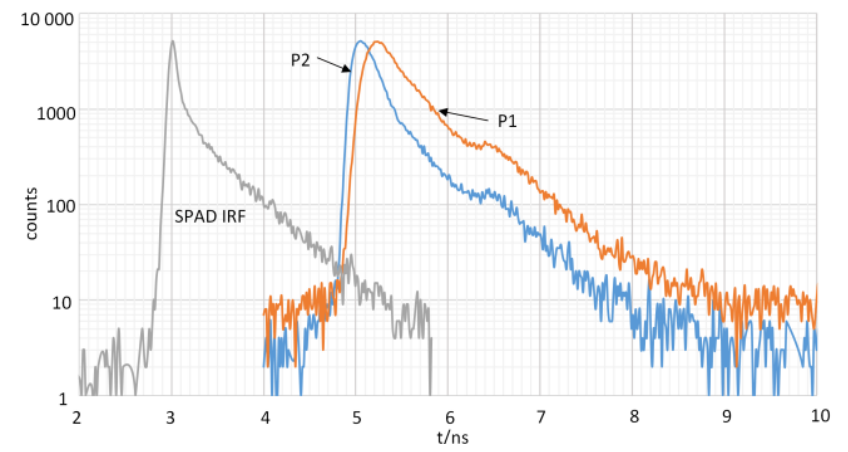

Fig. 6. IRF of the SPAD with the laser diode, and the DToFs of the phantoms 1 and 2.

\section{ACKNOWLEDGMENT}

The authors would like to thank Dr. Anssi Mäkynen and Mr. Harri Juttula providing the phantoms for TRDOI measurements.

\section{REFERENCES}

[1] A. Pifferi et al."New frontiers in time-domain diffuse optics, a review," Journal of Biomedical Optics, vol.21, no. 9, Art no. 091310, 2016.

[2] C. Niclass, M. Soga, H. Matsubara, M. Ogawa, M. Kagami, "A 0.18- $\mu$ m CMOS SoC for a 100-m-Range 10-Frame/s 200 x 96-Pixel Time-ofFlight Depth Sensor," IEEE Journal of Solid-State Circuits, vol. 49, no. 1, pp. 315-330, Jan. 2014.

[3] L. H. Brage et al.“ An 8x16-pixel 92 kSPAD time-resolved sensor with on-pixel $64 \mathrm{ps} 12 \mathrm{~b}$ TDC and $100 \mathrm{MS} / \mathrm{s}$ real-time energy histogramming in $0.13 \mu \mathrm{m}$ CIS technology for PET/MRI Applications," in IEEE Int. Solid-State Circuit Conference Dig. Tech . Papers, pp. 486-487, Jan. 2013.

[4] I. Nissinen et al. "A sub-ns time-gated CMOS single photon avalanche diode detector for Raman spectroscopy”, in Proc. IEEE ESSDERC, pp. 375-378,2011.

[5] D-U. Li et al." FPGA implementation of a video-rate fluorescence lifetime imaging system with a $32 \times 32$ CMOS single-photon avalanche diode array," IEEE International Symposium on Circuits and Systems, pp. 3082-3085, 24-27 May, 2009.

[6] T. Durduran, R. Choe, W. Baker and A. Yodh, 'Diffuse optics for tissue monitoring and tomography," Reports on Progress in Physics, vol. 73, no. 7, p. 076701,2010 .

[7] M.S. Patterson, B. Chance and B.C. Wilson,'Time resoved reflectance and transmittance for the non-invasive measurement of tissue optical properties," Appl. Opt., 28(12), pp.2331-2336, 1989.

[8] A. Dalla Mora et al.'Towards next-generation time-domain diffuse optics for extreme depth penetration and sensitivity," Biomedical Optics Express, Vol. 6, no. 5, pp. 1749-1760, 2015.

[9] S. Jahromi, J. Jansson, I. Nissinen, J. Nissinen and J. Kostamovaara, ”A Single Chip Laser Radar Receiver with a 9x9 SPAD Detector Array and a 10-Channel TDC," Proceedings of the ESSCIRC'15, Graz, Austria, pp. 364-367, Sep. 14-18, 2015.

[10] J. M. Pavia, M. Scandini, S. Lindner, M. Wolf and E. Charbon,"A 1x400 Backside-Illuminated SPAD Sensor With 49.7 ps Resolution, 30 pJ/Sample TDCs Fabricated in 3D CMOS Technology for Near-Infrared Optical Tomography," IEEE Journal of Solid-State Circuits, Vol. 50, no. 10, pp.2406-2418, 2015.

[11] D. Contini et al.,"Multichannel time-resolved tissue oximeter for functional imaging of the brain," IEEE Transactions on Instrumentation and Measurement, Vol. 55, no.1, pp. 85-90, 2006.

[12] L. Di Sieno et al.,'Miniaturized pulsed laser source for time-domain diffuse optics routes to wearable devices," Journal of Biomedical Optics, Vol. 22, no. 8, Art. no. 085004, 2017.

[13] J.M.T. Huikari et al. ," High-Energy Picosecond Pulse Generation by Gain Switching in Asymmetric Waveguide Structure Multiple Quantum Well Lasers," IEEE journal of Selected Topics in Quantum Electronics, vol. 21, no. 6, Art. no.1501206, Nov./Dec. 2015.

[14] J. Nissinen and J. Kostamovaara,'A High Repetition Rate CMOS Driver for High-Energy Sub-ns Laser Pulse Generation in SPAD-Based Timeof-Flight Range Finding," IEEE Sensors journal, Vol. 16, no. 6, pp.16281633, 2016.

[15] L. Pancheri, G-F Dalla Betta, L. Huf Campos Braga, H. Xu and D. Stoppa,"A Single-Photon Avalanche Diode test chip in 150nm CMOS technology," IEEE Conference on Microelectronic Test Structures, Udine, Italy, pp.161-164, March 24-27, 2014. 